\title{
The mid- and far-infrared colours of AGB and post-AGB stars ${ }^{\star}$
}

\author{
M. A. T. Groenewegen
}

Instituut voor Sterrenkunde, Katholieke Universiteit Leuven, Celestijnenlaan 200B, 3001 Leuven, Belgium

e-mail: groen@ster . kuleuven. be

Received 6 September 2005 / Accepted 27 October 2005

\section{ABSTRACT}

With the advent of space missions, like SPITZER and ASTRO-F, with sensitive detectors in the near- and mid-infra red covering a reasonable field-of-view and having a good spatial resolution, it will be possible to detect individual AGB stars in Local Group galaxies. The filters used by these missions are non-standard and different from mission to mission. In this paper, the colours of mass-losing AGB and post-AGB stars are calculated in the broad-band filters of the SPITZER and ASTRO-F missions, as well as Bessell $V, I$ and 2MASS $J, H, K$ to connect these results to existing ground-based data. The models are calculated for carbon- and oxygen-rich chemistry and cover different effective temperatures and dust compositions.

Key words. stars: circumstellar matter - stars: mass-loss - stars: AGB and post-AGB

\section{Introduction}

Asymptotic Giant Branch (AGB) and post-AGB stars are prominent emitters in the infrared (IR), firstly because of their low effective temperatures and secondly because of their mass loss rates, which can go up to $10^{-4} M_{\odot} \mathrm{yr}^{-1}$ for brief spells of time, in conjunction with dust formation. The importance of the mid-IR was first demonstrated by the results of the IRAS mission (Beichman et al. 1988) which discovered many masslosing AGB stars (e.g. van de Veen \& Habing 1988) in the solar neighbourhood but also luminous mass-losing AGB stars in the Large Magellanic Cloud (LMC) (see Reid et al. 1990; Reid 1991; Wood et al. 1992; Zijlstra et al. 1996; Loup et al. 1997), and Small MC (Whitelock et al. 1989; Groenewegen \& Blommaert 1998), at the limiting magnitudes of IRAS near $100 \mathrm{mJy}$ at 12 micron. When the ISO satellite was launched many of these mass-losing AGB stars discovered by IRAS in the MCs were followed up (e.g. Trams et al. 1999; van Loon et al. 1999), and new surveys in the MCs were conducted (Loup et al. 1999a,b), in particular using the ISOCAM instrument (Cesarsky et al. 1996; Blommaert et al. 2003) that had imaging filters covering 3 to 15 micron with a field-of-view of typically $1.5^{\prime}$ by $1.5^{\prime}$. In addition, the Galactic Centre region, where IRAS was severely confusion limited, was imaged during the ISOGAL survey (Omont et al. 2003), discovering many AGB stars (Omont et al. 1999).

IRAS and ISO showed the importance of the IR region, from the Near-IR to the Far-IR. The SPITZER mission (Werner et al. 2004), launched on 25 August 2003, and the ASTRO-F

* Appendix A is only available in electronic form at the CDS via anonymous ftp to cdsarc.u-strasbg.fr $(130.79 .128 .5)$ or via http://cdsweb.u-strasbg.fr/cgi-bin/qcat?]/A+A/448/181 mission (e.g. Pearson et al. 2004; Matsuhura et al. 2005), with a planned launch date of spring 2006, carry a range of imaging filters that cover the near-, mid- and far-IR. Due to an improved sensitivity and spatial resolution it is expected to resolve individual AGB stars in galaxies far beyond the MCs.

In the present work, series of dust radiative transfer models are presented that cover the spectral types, dust composition and mass loss rates seen for Galactic AGB stars. The flux-densities are computed in the SPITZER IRAC (Fazio et al. 2004) and MIPS (Rieke et al. 2004) filters, and the ASTRO-F IRC, MIR-S, MIR-L and FIS filters. For comparison to ground based data $V, I$ and $J, H, K$ magnitudes are also presented.

Section 2 presents the radiative transfer model and the inputs to it, including the various dust compositions considered. Section 3 gives details on the considered filter curves and the adopted flux-calibration. Section 4 presents the results, scaling laws to arbitrary luminosities and distances, and some caveats in the use of the results. Section 5 concludes by illustrating colour-magnitude diagrams for AGB stars in M31 and the Wolf-Lundmark-Melotte (WLM) galaxy.

Earlier results from this kind of dust modelling (in particular for the ISOCAM filters) were available as private communication and have been used in Blommaert et al. (2000), Ortiz et al. (2002) and Ojha et al. (2003).

\section{Inputs to the radiative transfer model}

The models have been calculated with a 1-dimensional dust radiative transfer (RT) code that solves the radiative transfer equation and the thermal balance equation in a self-consistent way (Groenewegen 1993, also see Groenewegen 1995). 

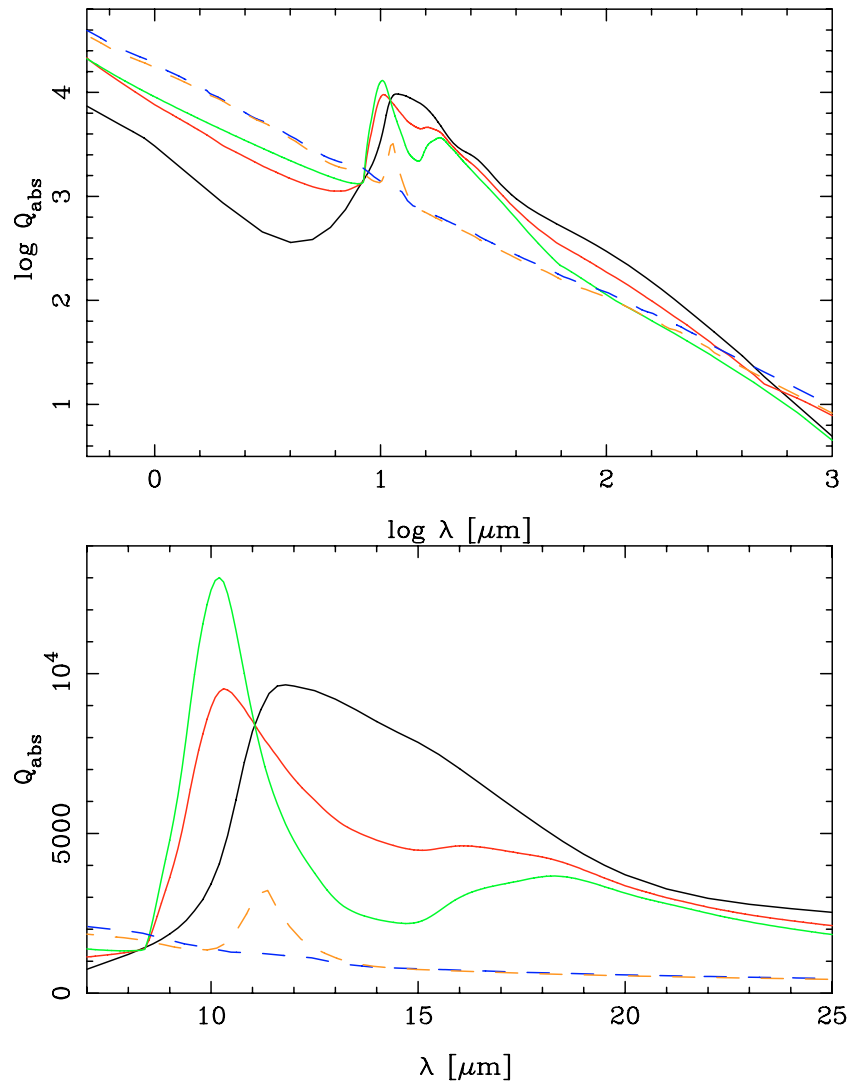

Fig. 1. The absorption efficiency for the five dust species considered here, plotted over the whole wavelength range (top panel), and the mid-IR. Dashed lines indicate 100\% AMC, and the combination of $85 \% \mathrm{AMC}+15 \% \mathrm{SiC}$, which peaks near $11.3 \mu \mathrm{m}$. The solid lines indicate $100 \%$ silicate (with a narrow feature that peaks near $10 \mu \mathrm{m}$ ), $40 \%$ silicate $+60 \% \mathrm{AlOx}$, and $100 \% \mathrm{AlOx}$ (with a broad feature that peaks near $12 \mu \mathrm{m})$.

Basic inputs to the model are the stellar luminosity $(L)$, distance $(d)$, photospheric spectrum, (total) mass loss rate, dust-to-gas ratio, (terminal) outflow velocity, dust condensation temperature $\left(T_{\mathrm{c}}\right)$ and composition of the dust.

The models have been calculated for (arbitrary) values of $L=3000 L_{\odot}, d=8.5 \mathrm{kpc}, v_{\infty}=10 \mathrm{~km} \mathrm{~s}^{-1}$, dust-to-gas $(\Psi)$ ratio $=0.005$, and no interstellar reddening. Scaling relations will be presented later.

Photospheric input spectrum for O-rich stars are taken from Fluks et al. (1994) for spectral types M0 $\left(T_{\text {eff }}=3850 \mathrm{~K}\right)$, M 6 $\left(T_{\text {eff }}=3297 \mathrm{~K}\right)$ and M $10\left(T_{\text {eff }}=2500 \mathrm{~K}\right)$. For C-rich stars the models by Loidl et al. (2001) for $T_{\text {eff }}=3600$ and $2650 \mathrm{~K}$ are considered ${ }^{1}$.

Several types of dust are considered that cover the main features observed in AGB stars. The absorption coefficients as a function of wavelength are displayed in Fig. 1. For dust around O-rich stars they are:

- 100\% Aluminium Oxide (AlOx; amorphous porous $\mathrm{Al}_{2} \mathrm{O}_{3}$ ), with optical constants from Begemann et al. (1997) and assuming a condensation temperature of $T_{\mathrm{c}}=1500 \mathrm{~K}$.

\footnotetext{
1 And with a C/O ratio of 1.1 .
}

- A combination of $60 \%$ AlOx and $40 \%$ Silicate (optical constants from David \& Pegourie 1995) and assuming a condensation temperature of $T_{\mathrm{c}}=1500 \mathrm{~K}$.

The dust species with 60 to $100 \%$ AlOx can explain the observed Spectral Energy Distributions (SEDs) and ISOCAM CVF 5-14 $\mu \mathrm{m}$ spectra in Galactic Bulge AGB stars which have mass loss rates up to $10^{-7} M_{\odot} \mathrm{yr}^{-1}$ (Blommaert 2003; Blommaert et al. 2005, in preparation).

- $100 \%$ silicate with $T_{\mathrm{c}}=1000 \mathrm{~K}$.

For dust around C-rich stars two species are considered:

- A combination of $85 \%$ Amorphous Carbon (AMC) and $15 \%$ Silicon Carbide ( $\mathrm{SiC}$ ) with optical constants from, respectively, Rouleau \& Martin (1991) for the AC1 species and $\alpha$-SiC from Pégourié (1988). A $T_{\mathrm{c}}$ of $1200 \mathrm{~K}$ is adopted.

- $100 \%$ AMC with a $T_{\mathrm{c}}$ of $1000 \mathrm{~K}$.

This range of AMC with zero to $15 \%$ percent $\mathrm{SiC}$ and the corresponding condensation temperatures can explain the SEDs and IRAS LRS spectra of the majority of (Galactic) C-stars (Groenewegen 1995; Groenewegen et al. 1998). For all dust species a uniform grain size of $0.1 \mu \mathrm{m}$ is adopted.

\section{Flux calibration}

In this paper the broad-band filters of the SPITZER and ASTRO-F missions are considered. Both contain filters in the near- and mid-IR which are ideal to identify mass losing stars. Filtercurves have been obtained from official websites ${ }^{2}$, and are reproduced in Fig. 2. The filter names and their central wavelengths are given in Table 1.

To connect this data to existing ground-based data Bessell $V, I$ and 2MASS $J, H, K$ magnitudes are also calculated, whose filter curves are also shown in Fig. 2.

The main output of the RT code is the flux emerging from the AGB star and its dust envelope. This flux is folded with the filter curves and flux-densities and magnitudes are calculated from (following the definition used by IRAS, ISOCAM and IRAC, see e.g. Blommaert et al. 2003):

$m_{\lambda}=-2.5 \log \left(\frac{\int\left(\lambda / \lambda_{0}\right) F_{\lambda} R_{\lambda} \mathrm{d} \lambda}{\int R_{\lambda} \mathrm{d} \lambda}\right)+m_{0}$.

The zero-points, $m_{0}$, are calculated from a reference spectrum for Vega calculated using the MARCS code (Decin, private communication). The resulting and adopted flux-densities for a zero-magnitude star are listed in Table 1.

\footnotetext{
2 http://ssc.spitzer.caltech.edu/mips/ spectral_response.html; http://ssc.spitzer.caltech.edu/irac/ spectral_response.html;
} http://www.ir.isas.jaxa.jp/ASTRO-F/Observation/ 

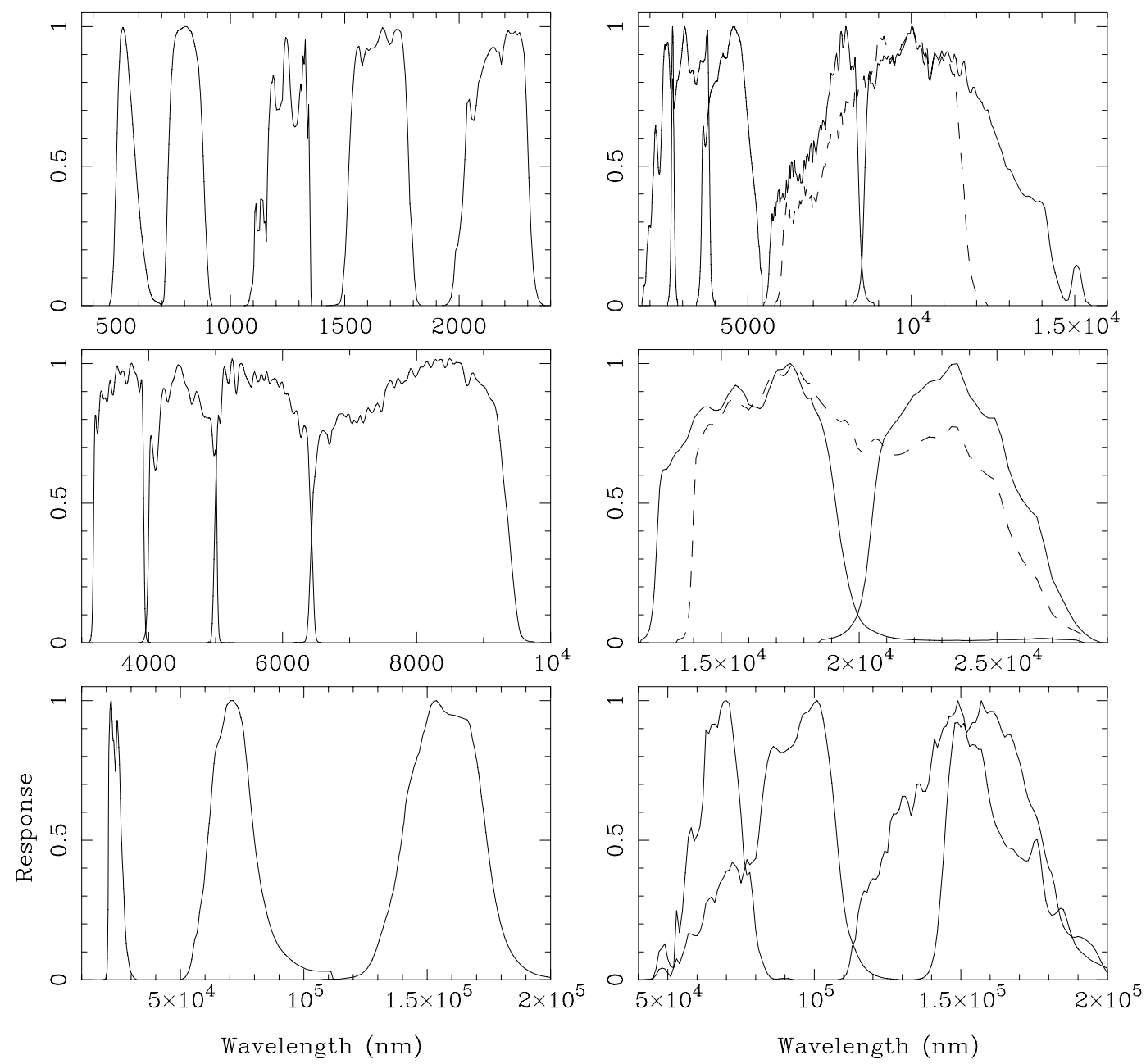

Fig. 2. Filter curves adopted: Bessell VI, 2MASS JHK (left top), Spitzer-IRAC (left middle), Spitzer-MIPS (left bottom), ASTRO-F IRC and MIR-S (right top), ASTRO-F MIR-L (right middle), and ASTRO-F FIS (right bottom).

Table 1. Adopted flux-densities for a zero-magnitude star.

\begin{tabular}{lrr}
\hline \hline Filter & $\lambda_{0}(\mu \mathrm{m})$ & Flux $(\mathrm{Jy})$ \\
\hline IRAC_3.6 & 3.55 & 278.8 \\
IRAC_4.5 & 4.49 & 179.6 \\
IRAC_5.8 & 5.73 & 118.4 \\
IRAC_8.0 & 7.87 & 63.5 \\
MIPS_24 & 24 & 7.23 \\
MIPS_70 & 70 & 0.791 \\
MIPS_160 & 160 & 0.190 \\
IRC_N2 & 2.69 & 651.4 \\
IRC_N3 & 3.06 & 323.5 \\
IRC_N4 & 4.55 & 199.8 \\
MIRS_S7 & 8.0 & 84.5 \\
MIRS_S9W & 10.04 & 57.7 \\
MIRS_S11 & 10.0 & 32.0 \\
MIRL_L15 & 17.5 & 17.6 \\
MIRL_L18W & 17.6 & 11.0 \\
MIRL_L24 & 23.55 & 7.45 \\
FIS_N60 & 60 & 0.935 \\
FIS_WIDE-S & 90 & 0.604 \\
FIS_WIDE-L & 140 & 0.194 \\
FIS_N160 & 160 & 0.166 \\
\hline
\end{tabular}

\section{Results for AGB and post-AGB stars}

As mentioned before, the results of the numerical code have already been used to successfully fit the SEDs of individual stars (Groenewegen 1994a,b, 1995, 1997; Groenewegen et al. 1995, 1997, 1998) and predict the colours of AGB stars (Blommaert et al. 2000; Ortiz et al. 2002; Ojha et al. 2003) and has been bench-marked against other codes (Ivezic et al. 1997). To illustrate the typical accuracies that can be achieved in fitting SEDs and colours, Fig. 3 shows a fit to the SED and the IRAS LRS spectrum of the Mira V Oph (cf. Fig. 33 in Groenewegen et al. 1998), but now with the $2650 \mathrm{~K}$ model atmosphere instead of a black-body in Groenewegen et al. (1998). The agreement is quite good over the whole wavelength range.

Figure 4 shows colour-colour diagrams of $(J-K)$ versus $(J-H),(J-[3.6])$ and $(J-[4.5])$ (the latter two being representative for $(J-L)$ and $(J-M)$, respectively). The data are the mean JHKLM magnitudes of carbon and oxygen-rich stars based on the monitoring of the light curves from Le Bertre (1992, 1993) and Taranova \& Shenavrin (2004). Only stars with magnitudes in all bands and without uncertain magnitudes were considered. 

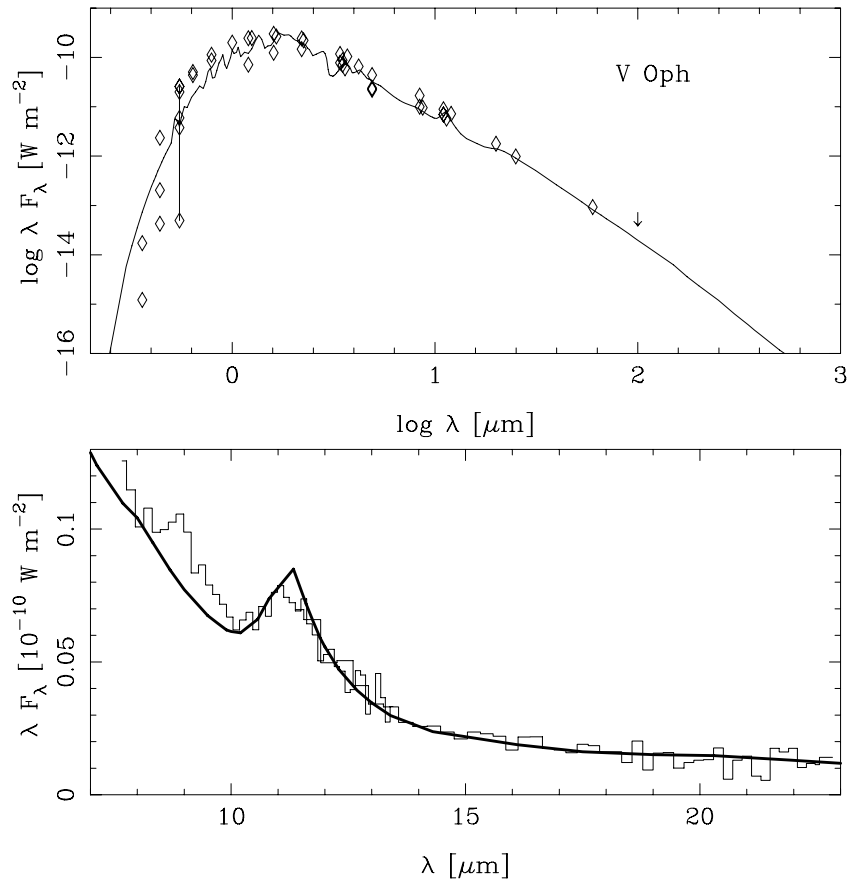

Fig. 3. Model fit to the SED and IRAS LRS spectrum of the carbon Mira V Oph (cf. Groenewegen et al. 1998). Used is the $2650 \mathrm{~K}$ model atmosphere, $L=4390 L_{\odot}, d=0.68 \mathrm{kpc}, \dot{M}=3.0 \times 10^{-8} M_{\odot} \mathrm{yr}^{-1}$, and a combination of $85 \% \mathrm{AMC}$ and $15 \% \mathrm{SiC}$.

Although such a comparison between models and data is not trivial because of the effects of reddening (although most sources are within $2.5 \mathrm{kpc}$ and reddening at these wavelengths should be small) and the fact that the IRAC [3.6] and [4.5] filters are not directly comparable to $L$ and $M$, it may nevertheless illustrate the overall correctness of the models, as suggested by the referee. Again, the overall agreement between models and data is quite good with most data points within 0.1 magnitude of any of the sequences.

The results of the models obtained in the present paper for the $V, I, J, H, K$, IRAC, MIPS and ASTRO-F magnitudes and fluxes are listed in Tables A.1 to A.26 for AGB stars and Tables A.27 to A.36 for post-AGB stars. Table A.1 is repeated as Table 2 to illustrate the content.

The AGB models have been calculated for mass loss rates up to a value resulting in a $(J-K)$ colour of about 10 , roughly the reddest known AGB stars (Groenewegen et al. 1998). For every set of input in terms of stellar input spectrum and dust composition there are two tables, listing, respectively, $V, I, J, H, K$ (in magnitudes) and the flux-densities (in mJy) for SPITZER IRAC and MIPS, and the flux-densities (in mJy) for the ASTRO-F IRC, MIR-S, MIR-L and FIS filters. Tables A.1-A.26 also list the mass loss rate and the dust optical depth, at $11.75 \mu \mathrm{m}$ for $\mathrm{AMC}+\mathrm{SiC}$ and $11.33 \mu \mathrm{m}$ for AMC, $11.75 \mu \mathrm{m}$ for AlOx and $\mathrm{AlOx}+$ silicate, and $10.20 \mu \mathrm{m}$ for silicate. Optical depths at other wavelengths can be estimated from Fig. 1. For the post-AGB models the dust temperature at the inner radius is listed instead of the mass loss rate. By decreasing this parameter the expansion of the dust envelope after cessation of mass loss at the end of the AGB can be simulated. The post-AGB tracks have only been calculated for the $100 \%$
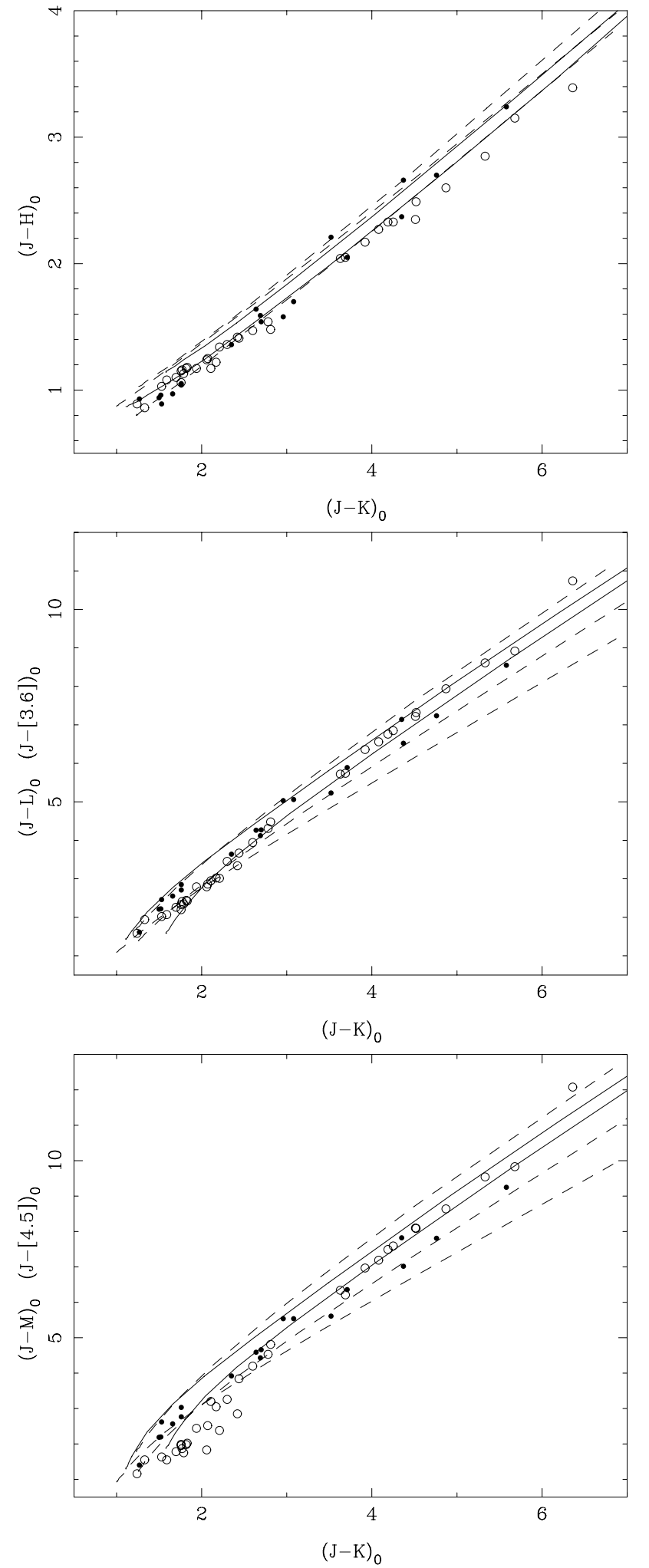

Fig. 4. Colour-Colour diagrams with $(J-K)$ plotted against $(J-H)$, $(J-[3.6])((J-L)$ for the observations), and $(J-[4.5])((J-M)$ for the observations). Carbon stars are plotted as open circles, oxygenrich stars as filled circles. Shown are model sequences for carbon stars (solid lines) for $T_{\text {eff }}=3600 \mathrm{~K}, 85 \% \mathrm{AMC}+15 \% \mathrm{SiC}$ dust, and $T_{\text {eff }}=$ $2650 \mathrm{~K}, 100 \% \mathrm{AMC}$ dust (the redder sequence), and oxygen-rich stars (dashed lines) for spectral type M0 and 100\% AlOx dust, M6 and 40\% silicate $+60 \%$ AlOx, and M10 and $100 \%$ silicate dust (from top to bottom for a given $(J-K))$. 
Table 2. Optical, NIR, IRAC and MIPS magnitudes and fluxes for a C-rich AGB star, $T_{\text {eff }}=2650 \mathrm{~K}, 85 \%$ AMC $+15 \%$ SiC. All tables are available on-line in Appendix A as Table A.1-A.36. In all tables $\dot{M}$ represents the mass loss rate in $M_{\odot} \mathrm{yr}^{-1}$, while $\tau$ represents the dust optical depth at the wavelengths listed in Sect. 4.

\begin{tabular}{rrrrrrrrrrrrrr}
\hline \hline$\dot{M}$ & $\tau$ & $V$ & $I$ & $J$ & $H$ & $K$ & 3.6 & 4.5 & 5.8 & 8.0 & 24 & 70 & 160 \\
\hline $0.10 \mathrm{E}-09$ & 0.000045 & 13.85 & 10.99 & 9.07 & 7.92 & 7.49 & 282.3 & 236.6 & 140.1 & 69.2 & 23.36 & 2.91 & 0.773 \\
$0.25 \mathrm{E}-09$ & 0.000113 & 13.85 & 10.99 & 9.07 & 7.92 & 7.49 & 282.5 & 236.9 & 140.3 & 69.4 & 23.42 & 2.92 & 0.776 \\
$0.50 \mathrm{E}-09$ & 0.000226 & 13.85 & 10.99 & 9.07 & 7.92 & 7.49 & 282.9 & 237.3 & 140.7 & 69.7 & 23.51 & 2.93 & 0.781 \\
$0.10 \mathrm{E}-08$ & 0.000452 & 13.85 & 11.00 & 9.07 & 7.92 & 7.49 & 283.6 & 238.1 & 141.5 & 70.5 & 23.71 & 2.97 & 0.790 \\
$0.25 \mathrm{E}-08$ & 0.001130 & 13.86 & 11.00 & 9.07 & 7.93 & 7.49 & 285.9 & 240.5 & 143.8 & 72.6 & 24.28 & 3.08 & 0.817 \\
$0.50 \mathrm{E}-08$ & 0.002259 & 13.87 & 11.01 & 9.08 & 7.93 & 7.49 & 289.7 & 244.6 & 147.7 & 76.1 & 25.24 & 3.26 & 0.863 \\
$0.10 \mathrm{E}-07$ & 0.004513 & 13.89 & 11.02 & 9.08 & 7.93 & 7.49 & 297.2 & 252.7 & 155.4 & 83.1 & 27.14 & 3.62 & 0.953 \\
$0.25 \mathrm{E}-07$ & 0.011247 & 13.96 & 11.07 & 9.11 & 7.95 & 7.48 & 319.2 & 276.5 & 178.3 & 103.7 & 32.77 & 4.69 & 1.223 \\
$0.50 \mathrm{E}-07$ & 0.022382 & 14.08 & 11.15 & 9.16 & 7.98 & 7.48 & 354.4 & 314.8 & 215.1 & 137.2 & 41.92 & 6.43 & 1.663 \\
$0.10 \mathrm{E}-06$ & 0.044342 & 14.31 & 11.31 & 9.25 & 8.03 & 7.49 & 418.4 & 385.8 & 284.2 & 200.4 & 59.44 & 9.75 & 2.498 \\
$0.25 \mathrm{E}-06$ & 0.108009 & 14.98 & 11.76 & 9.53 & 8.19 & 7.52 & 574.2 & 566.0 & 464.3 & 367.7 & 106.66 & 18.76 & 4.778 \\
$0.50 \mathrm{E}-06$ & 0.208266 & 16.02 & 12.47 & 9.96 & 8.46 & 7.61 & 747.2 & 786.7 & 698.1 & 592.6 & 173.15 & 31.57 & 8.023 \\
$0.10 \mathrm{E}-05$ & 0.393236 & 17.91 & 13.78 & 10.77 & 8.99 & 7.85 & 913.5 & 1054.0 & 1018.6 & 923.7 & 281.23 & 52.72 & 13.390 \\
$0.20 \mathrm{E}-05$ & 0.725089 & 21.26 & 16.12 & 12.23 & 9.97 & 8.40 & 955.8 & 1270.3 & 1375.0 & 1355.7 & 455.47 & 87.87 & 22.354 \\
$0.40 \mathrm{E}-05$ & 1.306390 & 26.92 & 20.19 & 14.77 & 11.71 & 9.47 & 775.9 & 1287.4 & 1647.3 & 1839.5 & 746.08 & 149.40 & 38.197 \\
$0.60 \mathrm{E}-05$ & 1.825759 & 31.81 & 23.78 & 16.99 & 13.21 & 10.42 & 587.2 & 1164.4 & 1699.8 & 2097.6 & 1002.97 & 206.71 & 53.101 \\
$0.10 \mathrm{E}-04$ & 2.756648 & 40.25 & 30.11 & 20.72 & 15.67 & 12.01 & 341.1 & 893.9 & 1602.9 & 2320.3 & 1458.59 & 315.02 & 81.643 \\
\hline
\end{tabular}

silicate and $100 \%$ AMC models, as it appears that dust with appreciable amounts of, respectively, AlOx and SiC only appear at lower mass loss rates, and not at the tip of the AGB. The model runs are such that the first entry for the post-AGB models corresponds to the same parameter set as for the last entry in the corresponding AGB model (e.g. Tables A.27-A.3, A.29-A.11, A.34-A.24) but listed as the result of an independent calculation. One can notice small differences of $\lesssim 1 \%$ in the fluxes and this is due to the accuracy of the RT model at these large optical depths.

\subsection{Scaling laws}

The fluxes listed in the tables scale with $(L / 3000) /(d / 8.5)^{2}$ (and the magnitudes as $-2.5 \log$ of this factor).

The spectral energy distribution is-for a given stellar input spectrum-determined only by the dust optical depth, defined by (Groenewegen 1993, 1995):

$$
\begin{aligned}
\tau_{\lambda} & =\int_{r_{\text {inner }}}^{r_{\text {outer }}} \pi a^{2} Q_{\lambda} n_{\mathrm{d}}(r) \mathrm{d} r \\
& =5.405 \times 10^{8} \frac{\dot{M} \Psi Q_{\lambda} / a}{R_{\star} v_{\infty} \rho_{\mathrm{d}} r_{\mathrm{c}}} \int_{1}^{x_{\max }} \frac{R(x)}{x^{2}} \mathrm{~d} x
\end{aligned}
$$

where $n_{\mathrm{d}}$ is the number density of dust particles, $x=r / r_{\mathrm{c}}$ and $\dot{M}(r)=\dot{M} R(x)$. The units are: the (present-day) mass loss rate at the inner radius $\dot{M}$ in $M_{\odot} \mathrm{yr}^{-1}, \Psi$ the dust-to-gas mass ratio, $Q_{\lambda} / a$ the absorption coefficient of the dust over the grain radius in $\mathrm{cm}^{-1}, R_{\star}$ the stellar radius in solar radii, $v_{\infty}$ the terminal velocity of the circumstellar envelope in $\mathrm{km} \mathrm{s}^{-1}, \rho_{\mathrm{d}}$ the dust grain density in $\mathrm{gr} \mathrm{cm}^{-3}, r_{\mathrm{c}}$ the inner dust radius in stellar radii and $x_{\max }$ the outer radius in units of $r_{\mathrm{c}}$. For the assumptions adopted in the present paper-a constant velocity and mass loss rate, and a outer radius much larger than the inner radius-the integral becomes unity.
This relation implies a necessary scaling when the expansion velocity and/or dust-to-gas ratio and/or luminosity are different from the nominal ones, and this scaling is like $\dot{M} \sim\left(v / 10 \mathrm{~km} \mathrm{~s}^{-1}\right)(0.005 / \Psi) \sqrt{L / 3000}$.

\subsection{Caveats}

My expectation is that these tracks might be useful to identify AGB stars in colour-colour and colour-magnitude diagrams that will become available when SPITZER and ASTRO-F results on external galaxies become available. Such a comparison might also be useful to provide indications of the chemical type and dust optical depth. However, when doing such comparisons some limitations of the models must be kept in mind.

- AGB stars are variable, usually of the Semi-Regular or Mira type. Mira variables can have pulsation amplitudes corresponding to 6-8 magnitudes in $V$, and up to $2.5 \mathrm{mag}$ in $K, 1.5 \mathrm{mag}$ in $M$ and $1.3 \mathrm{mag}$ in $N$ (e.g. Le Bertre 1992, 1993; Groenewegen et al. 1998). Little is known about the level of variability in the mid-IR, but for example CW Leo (IRC +10 216), with a full-amplitude in $K$ of $2.0 \mathrm{mag}$ (Le Bertre 1992), still has a full-amplitude at $850 \mu \mathrm{m}$ of 0.24 mag (Jenness et al. 2002).

- Other dust components may be present that are not taken into account ( $\mathrm{MgS}$ near $35 \mu \mathrm{m}$ in carbon stars, e.g. Hony et al. 2002, several crystalline silicate complexes near 23, 28, 33, 40 and $60 \mu \mathrm{m}$, see e.g. Molster et al. 2002) and that may influence some fluxes, especially below $\sim 60 \mu \mathrm{m}$.

- The dust shell may be non-spherical and scattered light might play a role.

- The models have been calculated assuming a constant mass loss rate. This may affect the far-IR colours (beyond $\sim 100 \mu \mathrm{m}$ ) either way. 
- The photospheric models used are appropriate for solar metallicities. This implies possible shifts in colour for low optical depths where the photosphere dominate the colours in systems of non-solar metallicity.

- The post-AGB model have been calculated under the assumption that the effective temperature and luminosity do not change over the time for the dust shell to drift away. For example, in the case of the $2650 \mathrm{~K}$ effective temperature C-rich central star, with a luminosity of $3000 L_{\odot}$ and $10 \mathrm{~km} \mathrm{~s}^{-1}$ expansion velocity it takes about 1100 year for the dust shell to expand to an inner dust radius temperature of $100 \mathrm{~K}, 8300 \mathrm{yr}$ to $50 \mathrm{~K}$, and $600000 \mathrm{yr}$ to $10 \mathrm{~K}$.

The transition timescale between the end of the AGB and the start of the PN phase (typically assumed to start at $10000 \mathrm{~K}$ ) is highly uncertain, and depends on the core mass (i.e. initial mass) of the star (the larger, the faster the evolution). Typical values are thought to be between 500 and 1500 years (e.g. Marigo et al. 2001, 2004).

This implies that only for inner dust radius temperatures above $\gtrsim 150 \mathrm{~K}$ the flow times scale is short enough for the assumption of constant luminosity and effective temperature likely to be valid.

- The final calibration may differ from the adopted one once the total throughput of the system has been established in-orbit.

\section{Discussion}

Dust radiative transfer models for (post-)AGB stars are presented from the optical to the far-infrared. The models are calculated in view of the (upcoming) results from SPITZER and (hopefully) ASTRO-F. They may be useful to identify AGB stars, and provide rough estimates of spectral type, luminosity and mass loss rate. I warn again, though, that once candidate AGB stars have been selected, the best estimates for mass loss rate, luminosity and dust composition can only be obtained by detailed fitting of the entire SED (and spectra if available) of individual stars.

As an illustration, Figs. 5 and 6 show some tracks in a colour-magnitude diagram for, respectively, SPITZER IRAC filters for an AGB star of $3000 L_{\odot}$ at a distance of $932 \mathrm{Kpc}$ (appropriate for WLM, McConnachie et al. 2005), and SPITZER MIPS filters for an AGB star of $3000 L_{\odot}$ at a distance of $785 \mathrm{Kpc}$ (appropriate for M31, McConnachie et al. 2005). WLM and M 31 are actual targets for those instruments according to the Spitzer Reserved Observations Catalog. One can observe that the tracks of the $\mathrm{C}$ - and O-rich mass-loss sequence largely overlap in [3.6-4.5] colour. Also the post-AGB tracks cover the same range in this colour. On the other hand, the [3.6] magnitude is a good indicator of the luminosity and the [3.6-4.5] colours of the mass loss rate. By comparison, in Fig. 6 C- and O-rich models separate, as well as AGB from post-AGB models. This illustrates the usefulness of a $70 \mu \mathrm{m}$ filter to trace post-AGB evolution. The MIPS $24 \mu \mathrm{m}$ filter traces the wing of the silicate $18 \mu \mathrm{m}$ feature and therefore the $24 \mu \mathrm{m}$ magnitude brightens quickly with the onset of mass-loss, so that the [24-70] colour initially becomes negative for O-rich stars. On the other hand, luminosity and mass loss rate are

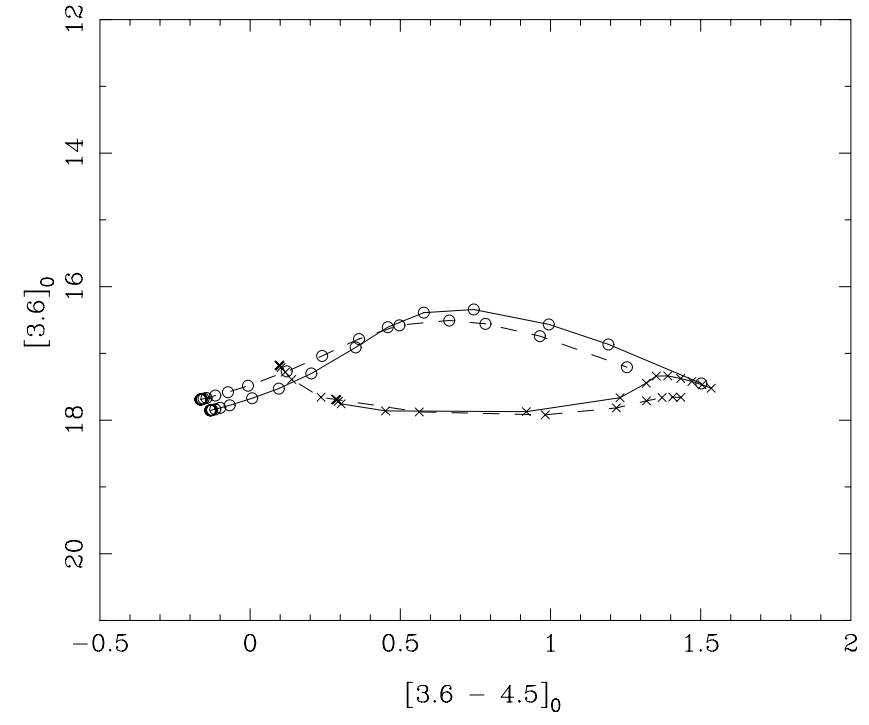

Fig. 5. IRAC colour-magnitude diagram for models with $3000 L_{\odot}$ at $932 \mathrm{Kpc}$. The following sequences of increasing mass loss are shown: Carbon-rich AGB star with $T_{\text {eff }}=3600 \mathrm{~K}$, and $85 \% \mathrm{AMC}+15 \% \mathrm{SiC}$ (circles \& solid line), Carbon-rich post-AGB star with $T_{\text {eff }}=2650 \mathrm{~K}$, and $100 \%$ AMC (crosses \& solid line), Oxygen-rich AGB star with $T_{\text {eff }}=3297 \mathrm{~K}$, and $60 \%$ silicate $+40 \%$ AlOx (circles \& dashed line), and oxygen-rich post-AGB star with $T_{\text {eff }}=2500 \mathrm{~K}$, and $100 \%$ silicate (crosses \& dashed line).

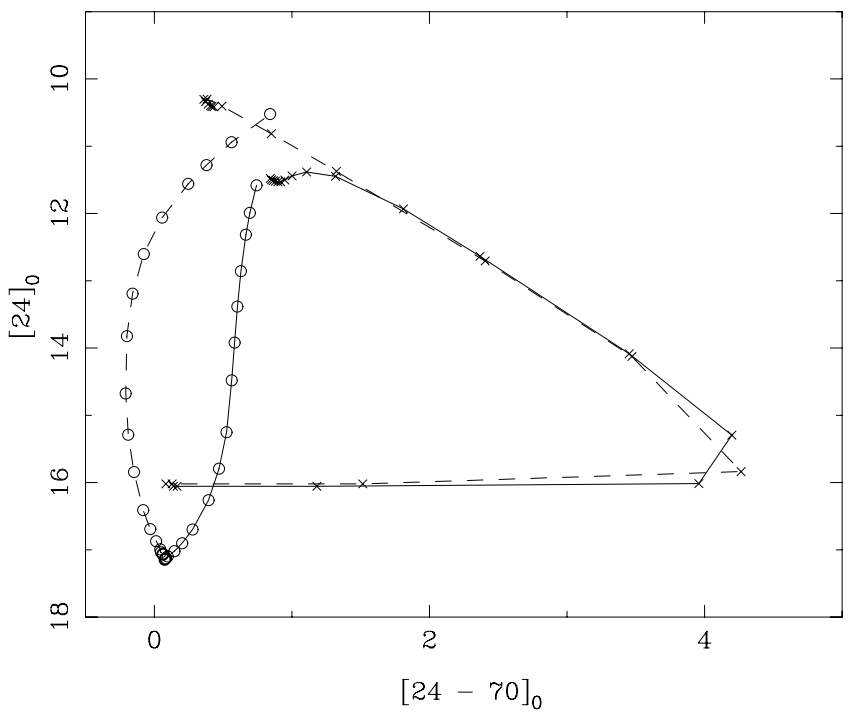

Fig. 6. MIPS colour-magnitude diagram for models with $3000 L_{\odot}$ at 785 Kpc. Model tracks as in Fig. 5.

difficult to discriminate as the AGB sequence is almost vertical in [24-70] colour.

Acknowledgements. I would like to thank Issei Yamamura (Institute of Space and Astronomical Science, ISAS, Kanagawa, Japan) for pointing out the availability of the ASTRO-F filter curves, and Leen Decin (K.U. Leuven) for providing a MARCS code model atmosphere for Vega. Joris Blommaert is thanked for reading an earlier version of the paper. 


\section{References}

Begemann, B., Dorschner, J., \& Henning, Th., et al. 1997, ApJ, 476, 199

Beichman, C. A., Neugebauer, G., Habing, H. J., et al. 1988, IRAS Explanatory Supplement, NASA RP-1190

Blommaert, J. A. D. L., Omont, A., The ISOGAL collaboration 2000 , Mem. Soc. Astron. It., 71, 623

Blommaert, J. A. D. L. 2003, in Exploiting the ISO Data Archive. Infrared Astronomy in the Internet Age, ed. C. Gry, S. Peschke, J. Matagne, P. Garcia-Lario, R. Lorente, \& A. Salama, ESA SP-511, 81

Blommaert, J. A. D. L., Siebenmoregen, R., Coulais, A., et al. 2003, The ISO handbook, volume II, ESA-SP 1262

Cesarsky, C. J., Abergel, A., Agnese, P., et al. 1996, A\&A, 315, L32

David, P., \& Pégourié, B. 1995, A\&A, 293, 833

Fazio, G., Hora, J. L., \& Allen, L. E. 2004, ApJS, 154, 1

Fluks, M. A., Plez, B., Thé, P. S., et al. 1994, A\&AS, 105, 311

Groenewegen, M. A. T. 1993, Ph.D. Thesis, Chapter 5, University of Amsterdam (available at http://www . ster . kuleuven . ac . be/ groen/thesis.ps.gz)

Groenewegen, M. A. T. 1994a, A\&A, 282, 115

Groenewegen, M. A. T. 1994b, A\&A, 290, 544

Groenewegen, M. A. T. 1995, A\&A, 293, 463

Groenewegen, M. A. T. 1997, A\&A, 317, 503

Groenewegen, M. A. T., \& Blommaert, J. A. D. L. 1998, A\&A, 332, 25

Groenewegen, M. A. T., Smith, C. H., Wood, P. R., Omont, A., \& Fujiyoshi, T. 1995, ApJ, 449, L119

Groenewegen, M. A. T., Oudmaijer, R. D., \& Ludwig, H.-G. 1997, MNRAS, 292, 686

Groenewegen, M. A. T., Whitelock, P. A., Smith, C. H., \& Kerschbaum, F. 1998, MNRAS, 293, 18

Hony, S., Waters, L. B. F. M., \& Tielens, A. G. G. M. 2002, A\&A, 390, 533

Ivezic, Z., Groenewegen, M. A. T., Me'shchikov, A., \& Szczerba, R. 1997, MNRAS, 291, 121

Jenness, T., Stevens, J. A., Archibald, E. N., et al. 2002, MNRAS, 336, 14

Le Bertre, T. 1992, A\&AS, 94, 377

Le Bertre, T. 1993, A\&AS, 97, 729

Loidl, R., Lançon, A., \& Jrgensen, U. G. 2001, A\&A, 371, 1065
Loup, C., Zijlstra, A. A., Waters, L. B. F. M., \& Groenewegen, M. A. T. 1997, A\&AS, 125, 419

Loup, C., Cioni, M.-R., Blommaert, J. A. D. L., et al. 1999a, in: The Universe as seen by ISO, ed. P. Cox, \& M. F. Kessler, ESA SP-427, 369

Loup, C., Josselin, E., Cioni, M.-R., et al. 1999b, IAU Symp., 191, ed. T. Le Bertre, A. Lebre, \& C. Waelkens, 561

Marigo, P., Girardi, L., Groenewegen, M. A. T., \& Weiss, A. 2001, A\&A, 378, 958

Marigo, P., Girardi, L., Weiss, A., Groenewegen, M. A. T., \& Chiosi, C. 2004, A\&A, 423, 995

Matsuhara, H., Shibai, H., Onaka, T., Usui, F., Adv. Space Res., accepted [arXiv: astro-ph/0507157]

McConnachie, A. W., Irwin, M. J., Ferguson, A. M. N., et al. 2005, MNRAS, 356, 979

Molster, F. J., Waters, L. B. F. M., \& Tielens, A. G. G. M. 2002, A\&A, 382,222

Ojha, D. K., Omont, A., Schuller, F., et al. 2003, A\&A, 403, 151

Omont, A., Ganesh, S., Alard, C., et al. 1999, A\&A, 348, 755

Omont, A, Gilmore, G. F., Alard, C., et al. 2003, A\&A, 403, 975

Ortiz, R., Blommaert, J. A. D. L., Copet, E., et al. 2002, A\&A, 388, 792

Pearson, C. P., Matsumoto, H. S., Murukami, H., et al. 2004, MNRAS, 347,1113

Pégourié, B. 1988, A\&A, 194, 335

Reid, N. 1991, ApJ, 382, 143

Reid, N., Tinney, C., \& Mould, J. 1990, ApJ, 348, 98

Rieke, G., Young, E. T., \& Engelbracht, C. W. 2004, ApJS, 154, 1

Rouleau, F., \& Martin, P. G. 1991, ApJ, 377, 526

Taranova, O. G., \& Shenavrin, V. I. 2004, PAZh, 30, 605

Trams, N. R., Van Loon, J. Th., Waters, L. B. F. M., et al. 1999, A\&A, 346,843

van der Veen, W. E. C. J., \& Habing, H. J. 1988, A\&A, 194, 125

van Loon, J. Th., Groenewegen, M. A. T., de Koter, A., et al. 1999, A\&A, 351, 559

Werner, M., Roellig, T., Low, F., et al. 2004, ApJS, 154, 1

Whitelock, P. A., Feast, M. W., Menzies, J. W., \& Catchpole, R. M. 1989, MNRAS, 238, 769

Wood, P. R., Whiteoak, J. B., Hughes, S. M. G., et al. 1992, ApJ, 397, 552

Zijlstra, A. A., Loup, C., Waters, L. B. F. M., et al. 1996, MNRAS, 279, 32 